\title{
The antibody response following a booster with either a 10- or 13-valent pneumococcal conjugate vaccine in toddlers primed with a 13-valent pneumococcal conjugate vaccine in early infancy
}

Trück, Johannes ; Jawad, Sena ; Goldblatt, David ; Roalfe, Lucy ; Snape, Matthew D ; Voysey, Merryn ; Pollard, Andrew J

\begin{abstract}
BACKGROUND Both the 13- and 10-valent pneumococcal conjugate vaccines (PCV-13; PCV10) are immunogenic and effective against vaccine-type pneumococcal disease when given to young children. However, limited data are available regarding the interchangeability of these 2 vaccines. METHODS UK children $(\mathrm{n}=178)$ who had previously been vaccinated with PCV-13 at 2 and 4 months were randomized to receive either a PCV-13 or a PCV-10 booster at 12 months of age. PCV-13 vaccine-type antipolysaccharide serum immunoglobulin G ( IgG) concentrations and opsonophagocytic assay titers were measured before and at 1 and 12 months following vaccination. The primary objective was to assess noninferiority of PCV-10 compared with PCV-13. RESULTS For 8 of the PCV-10 serotypes at least $97 \%$ of participants in both groups had IgG concentrations $0.35 \mu \mathrm{g} / \mathrm{mL}$ at 1 month after vaccination; inferior responses were seen for serotypes 5 and $9 \mathrm{~V}$ following the PCV-10 compared with the PCV-13 booster. Post booster geometric mean IgG concentrations and opsonophagocytic assay titers were significantly superior for most serotypes in PCV-13 compared with PCV-10 recipients, whereas similar or inferior responses were seen for serotypes 4, 18C, and 19F. Although some increase in antibody was seen in PCV-10 recipients against the serotypes 6A and 19A (serotypes that cross-react with $6 \mathrm{~B}$ and $19 \mathrm{~F}$ in PCV-10, respectively) at 1-month post booster, these responses were significantly lower than in the PCV13 group. CONCLUSIONS In PCV-13 primed infants, a PCV-10 booster is generally less immunogenic than a PCV-13 booster. For the 3 serotypes in PCV-10 with higher antigen content and/or conjugation to diphtheria or tetanus toxoid carrier proteins, higher or similar booster responses were seen in PCV-10 recipients. Although these findings suggest that responses are generally better with a PCV-13 booster among PCV-13 primed children, the clinical significance of these differences in immunogenicity is unclear.
\end{abstract}

DOI: https://doi.org/10.1097/INF.0000000000001180

Posted at the Zurich Open Repository and Archive, University of Zurich

ZORA URL: https://doi.org/10.5167/uzh-133455

Journal Article

Published Version

Originally published at:

Trück, Johannes; Jawad, Sena; Goldblatt, David; Roalfe, Lucy; Snape, Matthew D; Voysey, Merryn; Pollard, Andrew J (2016). The antibody response following a booster with either a 10- or 13-valent pneumococcal conjugate vaccine in toddlers primed with a 13-valent pneumococcal conjugate vaccine in early infancy. The Pediatric Infectious Disease Journal, 35(7):787-793.

DOI: https://doi.org/10.1097/INF.0000000000001180 


\title{
The Antibody Response Following a Booster With Either a 10- or 13-valent Pneumococcal Conjugate Vaccine in Toddlers Primed With a 13-valent Pneumococcal Conjugate Vaccine in Early Infancy
}

\author{
Johannes Trück, MD, DPhil, *† Sena Jawad, MSc, $\neq$ David Goldblatt, FRCPCH, PhD, $\$$ Lucy Roalfe, BSc, $\S$ \\ Matthew D. Snape, FRCPCH, * Merryn Voysey, M. Biostat, $\$$ and Andrew J. Pollard, FRCPCH, PhD*
}

\begin{abstract}
Background: Both the 13- and 10-valent pneumococcal conjugate vaccines (PCV-13; PCV-10) are immunogenic and effective against vaccine-type pneumococcal disease when given to young children. However, limited data are available regarding the interchangeability of these 2 vaccines.

Methods: UK children $(n=178)$ who had previously been vaccinated with PCV-13 at 2 and 4 months were randomized to receive either a PCV-13 or a PCV-10 booster at 12 months of age. PCV-13 vaccine-type antipolysaccharide serum immunoglobulin $\mathrm{G}(\mathrm{IgG})$ concentrations and opsonophagocytic assay titers were measured before and at 1 and 12 months following vaccination. The primary objective was to assess noninferiority of PCV-10 compared with PCV-13.
\end{abstract}

Results: For 8 of the PCV-10 serotypes at least $97 \%$ of participants in both groups had $\operatorname{IgG}$ concentrations $\geq 0.35 \mu \mathrm{g} / \mathrm{mL}$ at 1 month after vaccination;

Accepted for publication March 3, 2016.

From the *Oxford Vaccine Group, Department of Paediatrics, University of Oxford and the NIHR Oxford Biomedical Research Centre, Oxford, United Kingdom; †Paediatric Immunology, University Children's Hospital Zürich, Zürich, Switzerland; \$Nuffield Department of Primary Care Health Sciences, University of Oxford, Oxford, United Kingdom; and §Institute of Child Health, University College London, London, United Kingdom.

This study was sponsored by the University of Oxford and funded by the National Institute for Health Research (NIHR) Oxford Biomedical Research Centre and GSK Biologicals. The Oxford Vaccine Group acknowledges the support of the National Institute for Health Research Clinical Research Network. J.T. was supported by an ESPID Fellowship Award. The funders had no role in study design, data collection and analysis, decision to publish, or preparation of the manuscript.

Study concept and design: J.T., M.D.S. and A.J.P. Acquisition of data: J.T., D.G. Statistical analysis: S.J., J.T. and M.V. Interpretation of the data: J.T., M.D.S. and A.J.P. Drafting of the manuscript: J.T. Critical revision of the manuscript for important intellectual content: all authors.

A.J.P. has previously conducted studies on behalf of Oxford University funded by vaccine manufacturers, including the present study, but currently does not undertake industry funded clinical trials. A.J.P. chairs the UK Department of Health's (DH) Joint Committee on Vaccination and Immunisation (JCVI); the views expressed in this manuscript do not necessarily reflect the views of JCVI or DH. M.D.S. acts as chief or principal investigators for clinical trials conducted by the University of Oxford, sponsored by vaccine manufacturers, but receives no personal payments from them. M.D.S. has participated in advisory boards and industry sponsored symposia for vaccine manufacturers, but receives no personal payments for this study. M.D.S. and J.T. have received financial assistance from vaccine manufacturers to attend scientific conferences. D.G./L.R.'s laboratory performs contract serology and receives research funding from the manufacturers of pneumococcal vaccines. D.G. acts occasionally as an advisor to GSK and other vaccine manufacturers, is a NIHR Senior Investigator and is supported by the NIHR BRC at Great Ormond Street. The other authors have no conflicts of interest to disclose.

Address for correspondence: Johannes Trück, MD, DPhil, Oxford Vaccine Group, Department of Paediatrics, University of Oxford, Centre for Clinical Vaccinology and Tropical Medicine (CCVTM), Churchill Hospital, Old Road, Headington, Oxford, OX3 7LE, United Kingdom. E-mail: johannes. truck@paediatrics.ox.ac.uk.

Supplemental digital content is available for this article. Direct URL citations appear in the printed text and are provided in the HTML and PDF versions of this article on the journal's website (www.pidj.com).

Copyright (C) 2016 Wolters Kluwer Health, Inc. All rights reserved.

ISSN: 0891-3668/16/3507-0787

DOI: $10.1097 /$ INF.0000000000001180 inferior responses were seen for serotypes 5 and 9V following the PCV-10 compared with the PCV-13 booster. Post booster geometric mean IgG concentrations and opsonophagocytic assay titers were significantly superior for most serotypes in PCV-13 compared with PCV-10 recipients, whereas similar or inferior responses were seen for serotypes $4,18 \mathrm{C}$, and $19 \mathrm{~F}$. Although some increase in antibody was seen in $\mathrm{PCV}-10$ recipients against the serotypes 6A and 19A (serotypes that cross-react with $6 \mathrm{~B}$ and $19 \mathrm{~F}$ in PCV-10, respectively) at 1-month post booster, these responses were significantly lower than in the PCV-13 group.

Conclusions: In PCV-13 primed infants, a PCV-10 booster is generally less immunogenic than a PCV-13 booster. For the 3 serotypes in PCV-10 with higher antigen content and/or conjugation to diphtheria or tetanus toxoid carrier proteins, higher or similar booster responses were seen in PCV-10 recipients. Although these findings suggest that responses are generally better with a PCV-13 booster among PCV-13 primed children, the clinical significance of these differences in immunogenicity is unclear.

Key Words: interchangeability, pneumococcal conjugate vaccine, immunogenicity, children

(Pediatr Infect Dis J 2016;35:787-793)

$T$ he encapsulated bacterium Streptococcus pneumoniae commonly causes respiratory infections and may also cause severe infections, such as meningitis and sepsis. Pneumococcal proteinpolysaccharide conjugate vaccines (PCVs) were first routinely used in childhood immunization programs in children in the USA in 2000 and introduced as a 7-valent vaccine (PCV-7) into the UK infant immunization schedule in 2006, successfully reducing the burden of invasive pneumococcal disease in both countries (IPD). ${ }^{1-3}$ Newer PCVs with extended serotype coverage became available in 2009 including either an additional 3 (PCV-10) or 6 (PCV-13) serotypes. Both vaccines are immunogenic and effective against IPD when given to young children in a series of 3-4 vaccine doses. $^{3-6}$ Although both PCV-10 and PCV-13 share 10 serotypes, they differ substantially in the concentrations of polysaccharides, the method of conjugation, and the type and concentrations of the carrier proteins.

There are several potential advantages of interchanging PCVs and using PCV-10 in PCV-13 primed children. The use of a novel carrier protein such as nontypeable Haemophilus influenzaederived protein D in PCV-10, which is not closely related to an antigen included in any concurrently or previously administered routine vaccine minimizes the risk of interference related to the carrier protein although the effect is usually seen in primary vaccination. ${ }^{8}$ This in turn may enhance immune responses against the contained pneumococcal serotypes. In PCV-10, most serotypes are conjugated to protein $\mathrm{D}$, which has the potential to induce responses that may protect children against nontypeable Haemophilus influenzae disease through an immune response generated by the carrier 
protein,, 910 however this has yet to be demonstrated. Interchanging PCVs may help to improve our understanding of vaccine immunobiology, addressing unanswered questions in relation to the interaction of conjugate vaccines with the immune system ${ }^{11}$ and the poor booster responses with certain serotypes. ${ }^{7}$

This study aimed to investigate the potential of an alternative booster vaccine to be given to 12-month-old children who have been primed with PCV-13 in infancy. immunoglobulin $\mathrm{G}$ ( $\mathrm{IgG})$ concentrations and opsonophagocytic assay (OPA) titers for PCV13 serotypes were assessed before, 1 and 12 months following the booster. The safety and reactogenicity of the 12-month booster was assessed using diary cards containing parental reports of local and systemic reactions following vaccination.

\section{MATERIALS AND METHODS}

\section{Participants, Recruitment and Design}

The study was a randomized controlled clinical trial, which was open-labeled for participants and clinical trial staff, but blinded for laboratory staff. Ethical approval was obtained from the Oxfordshire Research Ethics Committee (reference number 11/ $\mathrm{SC} / 0473$ ) and the study was registered on Clinicaltrials.gov (registration number NCT01443416). Participants were recruited primarily by information booklets mailed out via the UK National Health Application and Infrastructure Services who are responsible for the central NHS patient database. Potential study participants were healthy 12-month-old children (range 50-58 weeks) at the time of enrolment, who were available for the entire study period and had received all primary vaccines according to the UK routine immunization schedule including 2 immunizations of PCV-13 at less than 6 months of age with a gap of at least 6 weeks between the 2 vaccinations. Absolute and temporary exclusion criteria are summarized in Table S1, Supplemental Digital Content 1, http://links.lww.com/ INF/C454. Following written informed consent, study participants were randomly allocated to receive a booster dose at 12 months of either PCV-13 (Prevenar 13/Prevnar 13, Pfizer Inc., New York, NY) or PCV-10 (Synflorix, GSK Vaccines, Rixensart, Belgium).

\section{Vaccines and Interventions}

Most serotypes contained in PCV-10 are conjugated individually to protein D (total dose 9-16 $\mathrm{g}$ ), a recombinant form of a lipoprotein of nontypeable $H$. influenzae; serotypes $18 \mathrm{C}$ and $19 \mathrm{~F}$ polysaccharides are conjugated to tetanus toxoid $(5-10 \mu \mathrm{g})$ and diphtheria toxoid (3-6 $\mu \mathrm{g})$, respectively (Table 1). PCV-13 contains saccharides from pneumococcal serotypes 3,6A and 19A in addition to PCV-10 serotypes, individually conjugated to crossreacting material $\left(\mathrm{CRM}_{197}\right.$, total dose of around $34 \mu \mathrm{g}$; Table 1). Both vaccines $(0.5 \mathrm{~mL})$ were administered intramuscularly using a $0.6 \times 25 \mathrm{~mm} 23$ gauge needle into the anterolateral aspect of either thigh. Combined $H$. influenzae type b/group $\mathrm{C}$ meningococcal vaccine and measles, mumps and rubella vaccine were given at 13 months but did not form part of the study evaluation.

\section{Laboratory Measurements}

Serum was separated within 24 hours at the study site and stored at $-20^{\circ} \mathrm{C}$. Antibody measurements were performed at the world health organization pneumococcal reference laboratory at University College London. Serum concentrations of total anticapsular IgG for PCV-13 serotypes were identified by ELISA method after absorption with $\mathrm{C}$ polysaccharide-containing cell wall extract and $22 \mathrm{~F}$ polysaccharide, and expressed as $\mu \mathrm{g} / \mathrm{mL}$. OPA titers against PCV-13 serotypes were measured using a multiplex opsonophagocytic assay. ${ }^{12}$ The lower limit of quantitation for all serotypes as established during the validation was $0.150 \mu \mathrm{g} / \mathrm{mL}$
TABLE 1. Pneumococcal Polysaccharide Concentrations and Serotype-specific Carrier Proteins Contained in PCV-10 and PCV-13

\begin{tabular}{|c|c|c|c|c|}
\hline \multirow[b]{2}{*}{ Serotype } & \multicolumn{2}{|c|}{ PCV-10* } & \multicolumn{2}{|c|}{ PCV-13† } \\
\hline & $\begin{array}{c}\text { Dose of } \\
\text { Polysaccharide } \\
(\mu \mathrm{g})\end{array}$ & $\begin{array}{l}\text { Carrier } \\
\text { Protein }\end{array}$ & $\begin{array}{c}\text { Dose of } \\
\text { Polysaccharide } \\
(\mu \mathrm{g})\end{array}$ & $\begin{array}{l}\text { Carrier } \\
\text { Protein }\end{array}$ \\
\hline 1 & 1 & Protein D & 2.2 & $\mathrm{CRM}_{197}$ \\
\hline 4 & 3 & Protein D & 2.2 & $\mathrm{CRM}_{197}^{197}$ \\
\hline 5 & 1 & Protein D & 2.2 & $\mathrm{CRM}_{197}^{197}$ \\
\hline $6 \mathrm{~B}$ & 1 & Protein D & 4.4 & $\mathrm{CRM}_{197}$ \\
\hline $7 \mathrm{~F}$ & 1 & Protein D & 2.2 & $\mathrm{CRM}_{197}$ \\
\hline $9 \mathrm{~V}$ & 1 & Protein D & 2.2 & $\mathrm{CRM}_{197}^{197}$ \\
\hline 14 & 1 & Protein D & 2.2 & $\mathrm{CRM}_{197}^{197}$ \\
\hline $18 \mathrm{C}$ & 3 & $\begin{array}{l}\text { Tetanus } \\
\text { toxoid }\end{array}$ & 2.2 & $\mathrm{CRM}_{197}^{197}$ \\
\hline $19 \mathrm{~F}$ & 3 & $\begin{array}{l}\text { Diphtheria } \\
\text { toxoid }\end{array}$ & 2.2 & $\mathrm{CRM}_{197}$ \\
\hline $23 \mathrm{~F}$ & 1 & Protein D & 2.2 & $\mathrm{CRM}_{197}$ \\
\hline 3 & & & 2.2 & $\mathrm{CRM}_{197}$ \\
\hline $6 \mathrm{~A}$ & & & 2.2 & $\mathrm{CRM}_{197}$ \\
\hline $19 \mathrm{~A}$ & & & 2.2 & $\mathrm{CRM}_{197}$ \\
\hline
\end{tabular}

$\dagger$ Contains $5 \mathrm{mM}$ succinate buffer and $0.125 \mathrm{mg}$ aluminum phosphate.

for IgG concentrations measured by ELISA and 8 for OPA titers. Values below the lower limit of quantitation were replaced with $0.075 \mu \mathrm{g} / \mathrm{mL}$ and 4 , respectively.

\section{Statistical Analysis}

Data were log-transformed before analysis and group summaries presented as geometric means with associated 2 sided, $95 \%$ confidence intervals (CI). In addition, the geometric mean foldrise for the serotype-specific antibody measurements was derived from the exponent of the within-person difference between the log-transformed individual assay result before and after the booster dose. The proportion of study participants achieving thresholds of $0.35 \mu \mathrm{g} / \mathrm{mL}$ for IgG concentrations, or 8 for OPA titers were calculated within each group and CIs computed using the binomial exact method. Similar analyses were performed to calculate proportions and $95 \%$ CI for rates of local and systemic reactions. Chi-squared test (or Fisher exact tests when expected counts were less than 5) were used to compare proportions of participants between groups. Two-sample $t$ tests or analysis of covariance was used to compare the continuous variables with adjustment for baseline values where appropriate. Comparisons between the 2 groups were carried out based on treatment allocation. Statistical analysis was performed using SAS version 9.3 (SAS Institute Inc., USA) and R. ${ }^{13}$

\section{Study Objectives}

The primary objective of this study was to assess whether PCV-10 is noninferior to PCV-13 using proportions of participants with IgG concentrations $\geq 0.35 \mu \mathrm{g} / \mathrm{mL}$ for PCV-10 serotypes 1 month following booster vaccination at 12 months of age with PCV-10 or PCV-13. Noninferiority of PCV-10 would be shown if the CI for the difference between groups (PCV-10 - PCV-13) did not include values less than $-10 \%$ (noninferiority margin). The sample size of 84 participants in each of the vaccine groups was calculated to allow assessment of noninferiority with a margin of $10 \%$ using $80 \%$ power and $2.5 \%$ significance level allowing for a $15 \%$ drop-out rate. During the course of the study, blood samples for less than $85 \%$ of participants were available at 13 months of age, therefore more study participants were recruited following amendment of the protocol. The intention to treat (ITT) population 
for immunogenicity consisted of all participants receiving a dose of PCV-10 or PCV-13 and providing a blood sample at age 13 months. Participants with predefined deviations from protocol were removed from the per-protocol (PP) population. The PP population was used for primary noninferiority analysis, with a sensitivity analysis performed on the ITT population. All other analyses were conducted on an ITT population; secondary objectives reported here included the immunogenicity and reactogenicity of the booster. Other secondary objectives that will be reported separately were the assessment of immediate pain at time of injection of the PCV booster and the investigation of antigen-specific peripheral blood memory $\mathrm{B}$ cells in response to vaccination.

\section{RESULTS}

A total of 178 children aged 11-13 months, from the Oxfordshire region, were recruited and randomized in a 1:1 ratio into 2 groups to receive 1 dose of either PCV-10 or PCV-13 at 12 months of age. Figure 1 shows the number of participants and their flow through the study. Baseline demographics of randomized participants are shown in Table 2.

One child was enrolled at age 11 months and 5 days, below the minimum age for inclusion, and was therefore excluded from the PP population (Fig. 1), but included in the ITT population. There were a number of protocol deviations related to the timing of

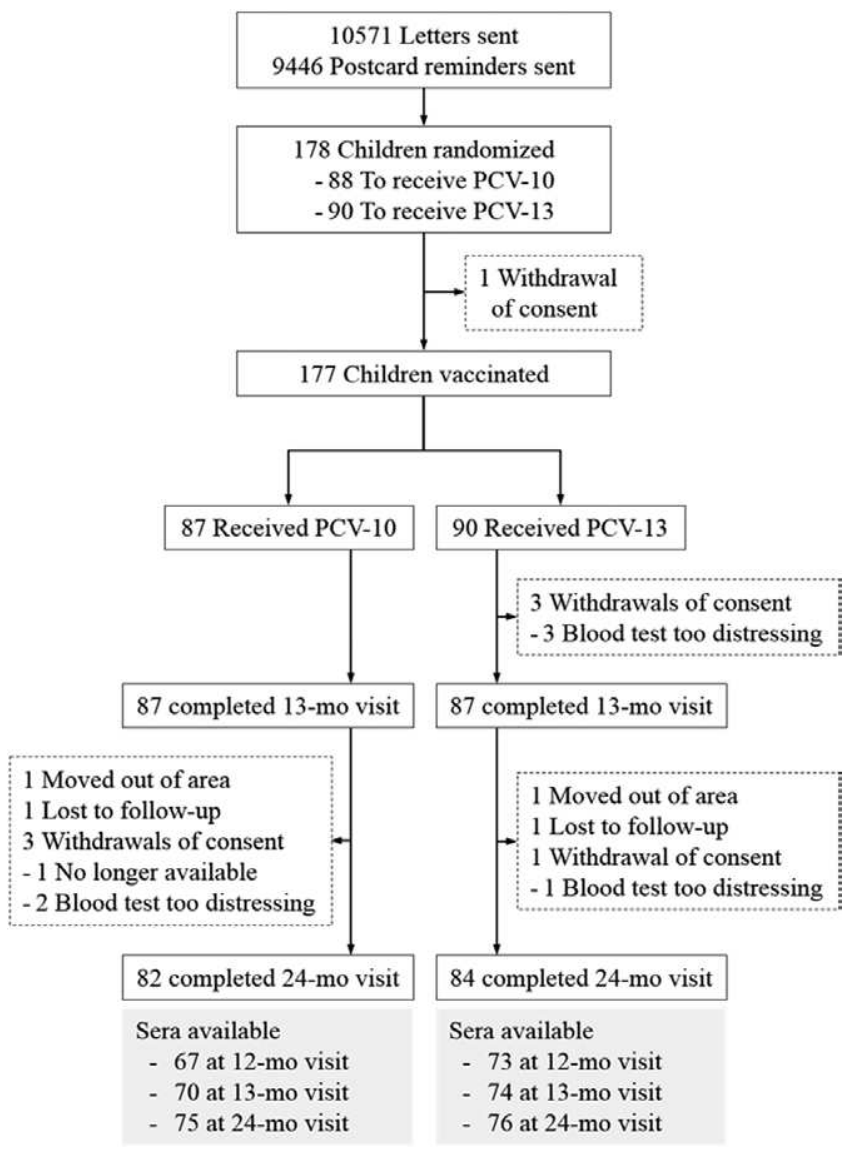

FIGURE 1. CONSORT diagram showing the flow through the study. One study participant receiving PCV-10 was excluded from the analysis of the primary objective because of major protocol violation resulting in $n=69$ in the PCV-10 group at the 13-month visit.
TABLE 2. Baseline Demographics by Group

\begin{tabular}{|c|c|c|}
\hline & PCV-10 (n = 87) & PCV-13 $(\mathrm{n}=90)$ \\
\hline & $\mathrm{N}(\%)$ & $\mathrm{N}(\%)$ \\
\hline \multicolumn{3}{|l|}{ Age (mos) } \\
\hline Mean (SD) [min-max] & $\begin{array}{c}12.6(0.4) \\
{[11.1-13.3]}\end{array}$ & $\begin{array}{c}12.7(0.4) \\
{[11.7-13.5]}\end{array}$ \\
\hline \multicolumn{3}{|l|}{ Sex } \\
\hline Male & $46(52.9)$ & $58(64.4)$ \\
\hline Female & $41(47.1)$ & $32(35.6)$ \\
\hline \multicolumn{3}{|l|}{ Ethnicity } \\
\hline $\begin{array}{l}\text { White Caucasian/ } \\
\text { European }\end{array}$ & $78(89.7)$ & $74(82.2)$ \\
\hline Other & $9(10.3)$ & $15(16.7)$ \\
\hline Unknown & $0(0)$ & $1(1.1)$ \\
\hline
\end{tabular}

SD indicates standard deviation.

visits, none of which were thought to significantly affect any of the outcome measures. Hence, none of these study participants were excluded from the PP population. There were 5 serious adverse events (febrile illness, suspected meningococcal sepsis, viral gastroenteritis, viral wheeze and febrile convulsion) reported during the study none of which were considered related to study vaccines and all participants recovered.

At baseline, there were significantly higher IgG GMC seen in the PCV-10 compared with the PCV-13 group for serotypes $19 \mathrm{~F}$ and 19A (Fig. 2). For serotype-specific geometric mean OPA titers (GMT), group differences were found only for serotype 3 with a significantly higher OPA GMT in PCV-10 compared with PCV-13 recipients (Fig. 3).

\section{Noninferiority Assessment of PCV-10 as an Alternative 12-month Booster (PP Population)}

Table 3 shows the results of the primary endpoint analysis. IgG responses to serotypes 5 and $9 \mathrm{~V}$ in the PCV-10 group were inferior to the PCV-13 group at 13 months when proportions of participants with serotype-specific IgG concentrations $\geq 0.35 \mu \mathrm{g} / \mathrm{mL}$ were compared between groups.

\section{Short-term Antibody Response to a Booster Dose of PCV-10 Compared With PCV-13 (ITT Population)}

One month following the booster vaccine, at least $97 \%$ of participants in each group had IgG concentrations $\geq 0.35 \mu \mathrm{g} / \mathrm{mL}$ for the serotypes they were vaccinated against with the exception of serotypes 5 and $9 \mathrm{~V}$ in the PCV-10 group. For these 2 serotypes, which are common to both PCV-10 and PCV-13, the proportions of participants with serotype-specific IgG above the threshold were significantly higher in PCV-13 compared with PCV-10 recipients (Figure S1 and Table S2, Supplemental Digital Content 1, http:// links.lww.com/INF/C454). In addition, these proportions were also higher in the PCV-13 group for 2 out of the 3 serotypes only included in PCV-13, namely serotypes 3 and 6A but not serotype 19A (Figure S1 and Table S2, Supplemental Digital Content 1, http://links.lww.com/INF/C454).

Post booster IgG GMC were significantly higher in the PCV-13 compared with the PCV-10 group for the majority of serotypes common to both vaccines $(1,5,6 \mathrm{~B}, 7 \mathrm{~F}, 9 \mathrm{~V}, 14$ and $23 \mathrm{~F})$ as well as for the serotypes only included in PCV-13 (3, 6A and 19A) whereas GMCs were significantly higher in PCV-10 compared with PCV-13 recipients only for serotypes 4, 18C and $19 \mathrm{~F}$ (Fig. 2; Table S4, Supplemental Digital Content 1, http://links. lww.com/INF/C454). 


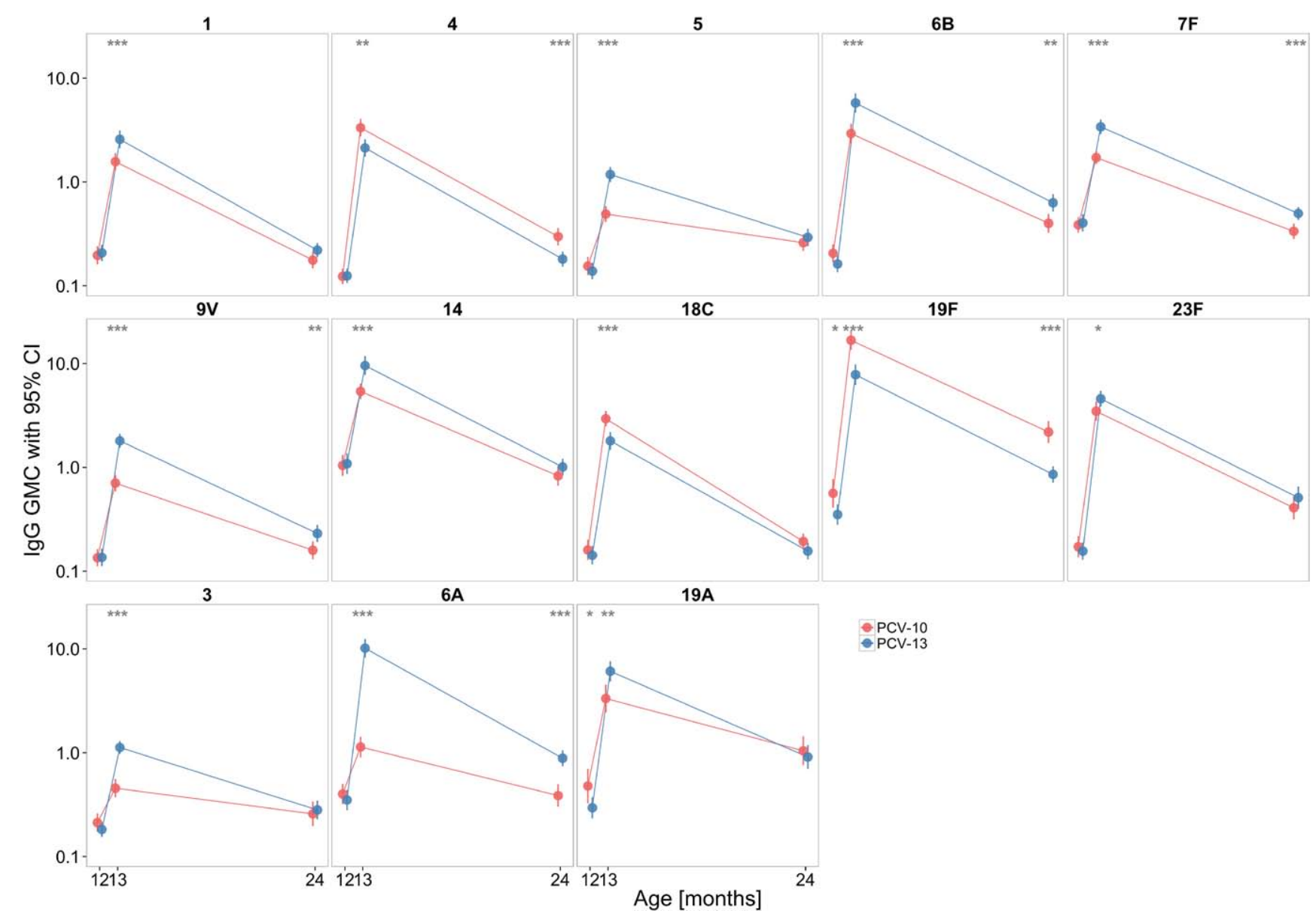

FIGURE 2. Serotype-specific IgG geometric mean concentrations by serotype and vaccine group at all 3 study time points. Groups were compared using independent samples $t$ tests using $\log _{10}$-transformed data with Satterthwaite correction for unequal variances and stars indicate the associated $P$ value $\left({ }^{* *}<0.001,{ }^{* *}<0.01\right.$ and $\left.{ }^{*}<0.05\right)$. full color

An analysis of covariance model that adjusted for baseline values, age, sex and ethnicity was used to investigate changes from baseline in IgG concentrations between groups. Significant increases in IgG concentrations were observed in both groups for all serotypes between 12 and 13 months of age and group differences from adjusted and unadjusted analyses were similar to differences observed in analysis of 13-month values alone (Table S6, Supplemental Digital Content 1, http:// links.lww.com/INF/C454).

Serotype-specific functional antibodies were assessed by OPA for all PCV-13 serotypes. One month following the booster, at least $98 \%$ of participants had titers $\geq 8$ and there were no observable group differences for all PCV-10 serotypes except for serotypes 1, 5 and $9 \mathrm{~V}$; for these serotypes the proportion of participants with OPA titers $\geq 8$ was higher in the PCV-13 than the PCV-10 group. These proportions were also higher for all 3 serotypes (3, 6A and 19A) only contained in PCV-13 (Table S7 and Figure S2, Supplemental Digital Content 1, http://links.lww.com/INF/C454).

Significantly higher post booster GMT were seen in the PCV-13 than the PCV-10 group for most serotypes common to both vaccines as well as for all 3 serotypes only included in PCV-13. For serotypes 4 and $18 \mathrm{C}$, there were no significant differences between the groups and for serotype $19 \mathrm{~F}$, the OPA response was significantly higher in the PCV-10 than the PCV-13 group (Fig. 3; Table S9, Supplemental Digital Content 1, http://links.lww.com/INF/C454).
For both study groups, significant increases in OPA titers for all serotypes with the exception of serotype 3 in the PCV-10 group were seen between 12 and 13 months of age (Table S11, Supplemental Digital Content 1, http://links.lww.com/INF/C454). Analyses of change from baseline to 13 months in OPA titers with adjustment for baseline values, age, sex and ethnicity gave similar results to unadjusted analyses of 13 month values although group differences in immunogenicity for serotypes 1 and 19F were no longer observed (Table S11, Supplemental Digital Content 1, http://links. lww.com/INF/C454).

\section{One-year Antibody Persistence Following a Booster Dose of PCV-10 Compared With PCV-13 (ITT Population)}

Group differences in proportions above the IgG or OPA thresholds were observed for a number of serotypes at 24 months of age, that is, 12 months following the booster. These proportions were similarly greater for both measurements for PCV-13 compared with PCV-10 recipients for serotypes $6 \mathrm{~B}, 7 \mathrm{~F}$ and $6 \mathrm{~A}$ and lower for serotype 19F (Figures S1 and S2, Supplemental Digital Content 1, http://links.lww.com/INF/C454.).

When persistence of $\operatorname{IgG}$ concentrations and OPA titers were assessed at 24 months of age, significant differences remained between the groups. IgG GMCs and OPA GMTs were greater in PCV-13 compared with PCV-10 recipients for serotypes $6 \mathrm{~B}, 7 \mathrm{~F}$, 
1

45

$5-6 B$

7F

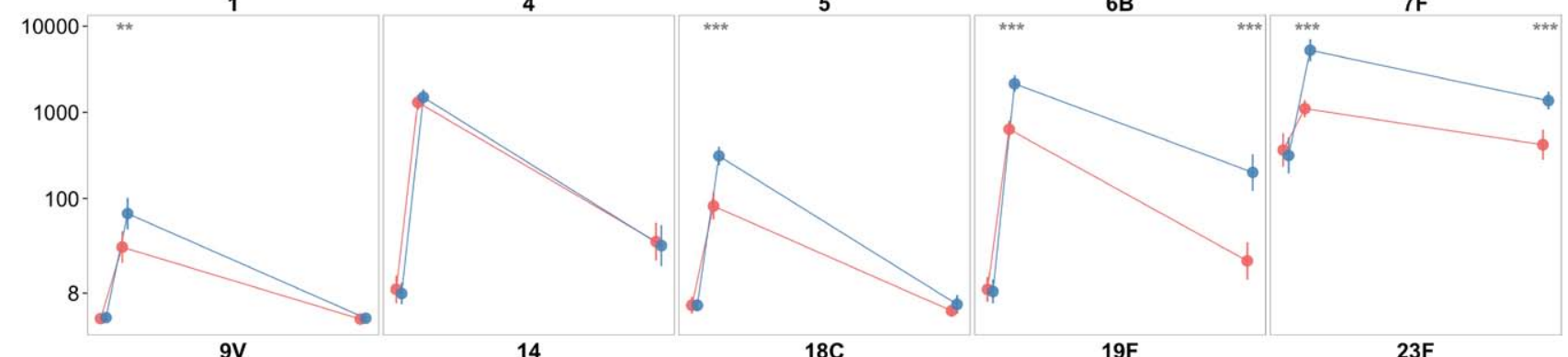

$9 \mathrm{~V}-14$

$18 \mathrm{C}$

$19 \mathrm{~F}$

$23 \mathrm{~F}$



3

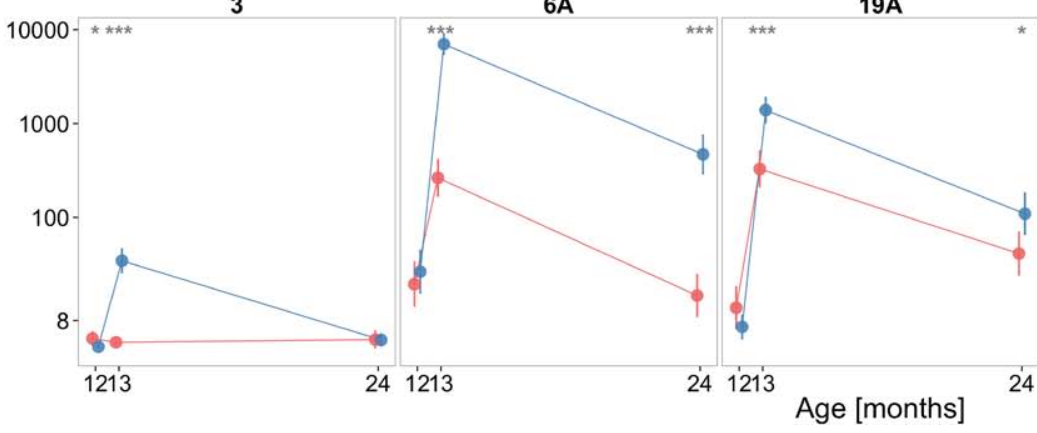

- PCV -10

Age [months]

FIGURE 3. Serotype-specific geometric mean OPA titers by serotype and vaccine group at all 3 study time points. Groups were compared using independent samples $t$ tests using $\log _{10}$-transformed data with Satterthwaite correction for unequal variances and stars indicate the associated $P$ value $\left({ }^{* *}<0.001,{ }^{* *}<0.01\right.$ and $\left.{ }^{*}<0.05\right)$. full color

$9 \mathrm{~V}$ and $6 \mathrm{~A}$, whereas both measurements were significantly higher in the PCV-10 compared with the PCV-13 group for serotype 19F (Figs. 2 and 3; Tables S5 and S10, Supplemental Digital Content 1, http://links.lww.com/INF/C454.).

When analyses of change from baseline to 24 months were adjusted for baseline antibody, age, sex and ethnicity, group differences were similar to unadjusted analyses (Tables S12 and S13, Supplemental Digital Content 1, http://links.lww.com/INF/C454.) with a significant decline in serotype-specific IgG antibodies and OPA titers from 13 to 24 months for all serotypes except for OPA titers against serotype 3 in the PCV-10 group (not shown).

\section{Reactogenicity}

The reactogenicity profile of the booster vaccination was similar regardless of whether participants had received PCV-10 or PCV-13 (Table S14, Supplemental Digital Content 1, http://links. lww.com/INF/C454). Local reactions such as redness, hardness and swelling were either absent or mild in most cases. Moderate or severe localized pain was reported by almost $13 \%$ of parents of study participants across both groups; irritability, drowsiness and decreased appetite were recorded in 53\%, 29\% and $29 \%$ of participants of both groups combined, respectively (Table S15, Supplemental Digital Content 1, http://links.lww.com/INF/C454). Low-grade fever $\left(38-39^{\circ} \mathrm{C}\right)$ was noted in $4 \%$ of participants and 4 children $(2 \%)$ had a temperature of $>39^{\circ} \mathrm{C}$ in the first 4 days following booster vaccination (Table S15, Supplemental Digital
Content 1, http://links.lww.com/INF/C454). The majority of these reported adverse effects of vaccination were short-lived lasting 1-3 days (Table S16, Supplemental Digital Content 1, http://links.lww. com/INF/C454).

\section{DISCUSSION}

This is the first study to investigate the interchangeability of PCV-10 and PCV-13 as a 12-month booster in children primed with PCV-13; the immune response to a PCV-13 booster given to children primed with PCV-10 was not assessed in the study. A robust antibody response was induced by both vaccines at 1 year of age when administered to PCV-13 primed children. For the primary objective, PCV-10 was noninferior to PCV-13 for 8 of the PCV-10 serotypes. However, there were $22 \%$ and $10 \%$ less study participants with IgG concentrations $\geq 0.35 \mu \mathrm{g} / \mathrm{mL}$ against serotypes 5 and $9 \mathrm{~V}$, respectively, in the PCV-10 than the PCV-13 group (Table 3). Children with IgG concentrations $\geq 0.35 \mu \mathrm{g} / \mathrm{mL}$ following 3 infant doses have previously been described as having clinical protection against IPD. ${ }^{14}$ However, the significance of this threshold in a situation where post booster immune responses are assessed is unknown and may underestimate the true differences between the 2 studied vaccines, as antibody responses are generally higher following booster doses and with older age. ${ }^{15,16}$ In addition, a quantitatively and qualitatively better vaccine response is thought to have greater potential to prevent carriage of vaccine serotypes and therefore 
TABLE 3. Proportion of Participants With IgG Antibody Concentrations $\geq 0.35 \mu \mathrm{g} / \mathrm{mL}$ by Serotype and Vaccine Group at 13 Months and Difference Between Groups (PP Population)

\begin{tabular}{|c|c|c|c|c|c|}
\hline \multirow[b]{2}{*}{ Serotype } & \multicolumn{2}{|c|}{ PCV-10 $(\mathrm{n}=69)$} & \multicolumn{2}{|c|}{ PCV-13 $(\mathrm{n}=74)$} & PCV-10 - PCV-13 \\
\hline & $\mathrm{N}$ & $\begin{array}{c}\text { Proportion } \\
\text { IgG } \geq 0.35 \mu \mathrm{g} / \mathrm{mL} \\
\quad(95 \% \mathrm{CI})\end{array}$ & $\mathrm{N}$ & $\begin{array}{c}\text { Proportion } \\
\text { IgG } \geq 0.35 \mu \mathrm{g} / \mathrm{mL} \\
(95 \% \mathrm{CI})\end{array}$ & $\begin{array}{c}\text { Difference } \\
(95 \% \mathrm{CI})\end{array}$ \\
\hline 1 & 69 & $0.986(0.922,1.000)$ & 74 & $0.987(0.927,1.000)$ & $-0.001(-0.040,0.038)$ \\
\hline 4 & 69 & $1.000(0.948,1.000)$ & 74 & $0.987(0.927,1.000)$ & $0.014(-0.013,0.040)$ \\
\hline 5 & 69 & $0.739(0.619,0.837)$ & 74 & $0.960(0.886,0.992)$ & $-0.220(-0.336,-0.105)$ \\
\hline $6 \mathrm{~B}$ & 69 & $0.971(0.899,0.997)$ & 73 & $1.000(0.951,1.000)$ & $-0.029(-0.070,0.012)$ \\
\hline $7 \mathrm{~F}$ & 69 & $0.986(0.922,1.000)$ & 74 & $1.000(0.951,1.000)$ & $-0.015(-0.043,0.014)$ \\
\hline $9 \mathrm{~V}$ & 69 & $0.884(0.784,0.949)$ & 74 & $0.987(0.927,1.000)$ & $-0.102(-0.184,-0.021)$ \\
\hline 14 & 69 & $1.000(0.948,1.000)$ & 74 & $1.000(0.951,1.000)$ & $0.000(0.000,0.000)$ \\
\hline $18 \mathrm{C}$ & 69 & $1.000(0.948,1.000)$ & 74 & $0.973(0.906,0.997)$ & $0.027(-0.011,0.065)$ \\
\hline $19 \mathrm{~F}$ & 69 & $1.000(0.948,1.000)$ & 74 & $0.987(0.927,1.000)$ & $0.014(-0.013,0.040)$ \\
\hline $23 \mathrm{~F}$ & 63 & $1.000(0.943,1.000)$ & 74 & $1.000(0.951,1.000)$ & $0.000(0.000,0.000)$ \\
\hline
\end{tabular}

Bold indicates significant group differences (serotypes 5 and $9 \mathrm{~V}$ ).

providing herd protection. This is most relevant for some serotype ${ }^{17}$ and young children who are responsible for pneumococcal transmission. ${ }^{18}$ Therefore, the vaccine with the best post booster immune response may help providing better indirect protection for unvaccinated individuals.

When post booster serotype-specific IgG GMC and OPA GMT were considered, PCV-13 was more immunogenic for the majority of serotypes common to both vaccines. There are no published large head-to-head clinical trials comparing PCV-10 and PCV13. In a recent small study in which children were vaccinated with either PCV-10 or PCV-13 at 2, 3, 4 and 11 months of age, serotypespecific antibodies, plasma and memory B cells against PCV-10 serotypes were investigated and compared between the groups before as well as 7-9 days and 1 month following the booster dose. ${ }^{19,20}$ This study showed statistically superior post booster $\operatorname{IgG}$ responses in the PCV-13 group to the majority of serotypes common to both vaccines, including serotype $19 \mathrm{~F}$, but not serotypes 4 and 18C. In the same study, 1 week post booster OPA GMTs were higher for $2(9 \mathrm{~V}$ and $23 \mathrm{~F}$ ) of the 10 common serotypes in PCV-13 compared with PCV-10 recipients. For no serotype were post booster antibody responses in the PCV-13 group significantly lower than in the PCV-10 group. ${ }^{20}$ These findings are partly in contrast to our study in which responses to serotype $19 \mathrm{~F}$ were lower in PCV-13 recipients for both IgG levels and OPA titers at 1 and 12 months following the booster.

In our study, only responses against serotypes 4, 18C and 19F were consistently similar or statistically superior (19F) at 13 months in the PCV-10 compared with the PCV-13 group. Interestingly, these 3 serotypes are contained in PCV-10 in a higher concentration than the other serotypes ( 3 vs. $1 \mu \mathrm{g}$ ) and also compared with the same serotypes in PCV-13 (3 vs. $2.2 \mu \mathrm{g}$ ). In addition, PCV-10 serotypes $18 \mathrm{C}$ and $19 \mathrm{~F}$ are conjugated to a non-protein $\mathrm{D}$ carrier protein - tetanus and diphtheria toxoid, respectively — and both these antigens are included in previously administered routine childhood vaccines. This may also partly explain the better antibody response to these 2 serotypes compared with other serotypes in $\mathrm{PCV}-10$ recipients as carrier priming would have already happened through these other vaccines. The enhanced immunogenicity of serotype 19F compared with other serotypes in the PCV-10 group may also be explained by the overall very similar structure of the 2 carrier proteins to which serotype $19 \mathrm{~F}$ is conjugated in PCV-10 (diphtheria toxoid) and PCV-13 $\left(\mathrm{CRM}_{197}\right){ }^{21}$ Although there are no immunogenicity studies directly comparing PCV-10 with PCV-13, antibody responses to PCV-10 have previously been compared with those elicited by PCV-7.22 Vesikari et $\mathrm{al}^{22}$ showed that post booster antibody responses in participants who had exclusively received either vaccine were superior in the PCV-7 group for the majority of PCV-7 serotypes with the exception of serotypes $18 \mathrm{C}$ (similar) and 19F (inferior) indicating that PCV-7 is generally more immunogenic than PCV-10. In the same study, antibody responses were largely similar between the group of children who had exclusively received PCV-7 and those who had been primed with PCV-7 followed by a booster dose of PCV-10. These findings suggest that similar antibody responses can be achieved when switching from one PCV to another containing similar capsular serotypes despite a difference in carrier proteins. However, Vesikari et $\mathrm{a}^{22}$ also demonstrated that there were markedly lower antibody responses to serotypes 1, 5 and 7F (not included in PCV-7) in children in the PCV-7/PCV-10 compared with the PCV-10/PCV-10 group indicating that serotype-specific priming even when performed with a different vaccine and carrier protein is essential for obtaining booster responses. In a case-control study performed in Canada, the effectiveness against all IPD caused by PCV-13 serotypes of a mixed PCV schedule consisting of 2 doses of PCV-10 followed by 1 dose of PCV13 was similar to alternative schedules in which children were only vaccinated with PCV-10 or PCV-13. ${ }^{4}$ Although this study suffered from a small sample size, and therefore the lack of significant differences between the vaccine schedules may not represent equivalence, these findings suggest that interchanging PCVs does not per se result in inferior protection compared with schedules using a single type of PCV. The combination of priming with PCV-13 and boosting with PCV-10 was also not assessed in the study.

In a previous study examining the effect of a booster dose of the 23-valent plain pneumococcal conjugate vaccine (PPV-23) given to 12-month-old children, researchers found that a good immune response could be elicited by PPV-23 in children primed with PCV-7. However, these children subsequently showed hyporesponsiveness when challenged with a $20 \%$ PPV- 23 dose, ${ }^{23}$ suggesting that measuring antibody alone may not be sufficient when comparing the immunogenicity of different polysaccharide-containing vaccines. Further assessments of the immune response such as antibody persistence and memory B cells may be needed to better capture the immune response to a vaccine booster.

For the 3 serotypes that are exclusive to PCV-13 (3, 6A and 19A), not only PCV-13 but also PCV-10 recipients showed an increased antibody response following booster vaccination, which may represent generation of cross-reactive antibodies through vaccination with the related serotypes 6B and 19F. Not surprisingly, the antibody responses against these 3 serotypes were greatly lower in the PCV-10 compared with the PCV-13 group (Figs. 2 and 3). 
In this study, antibody persistence up to 1 year following the booster was assessed using both serotype-specific IgG antibody concentrations and OPA titers against PCV-13 serotypes. Over the 12-month period following the booster, there was a rapid decline in antibody in both groups. IgG levels and OPA titers at 24 months were largely determined by the amount of antibody detected 1 month post booster at 13 months of age, which has been previously demonstrated where the same PCV was used for both priming and boosting. ${ }^{24}$ These findings suggest that boosting with a different vaccine containing the same capsular serotypes, albeit conjugated to different carrier proteins, induces an immune response that is not only driven by short-lived extrafollicular B cells but also the generation of long-lived plasma cells, a hallmark of immune memory.

\section{CONCLUSIONS}

Our study showed that a booster dose of PCV-10 at 12-months of age is generally less immunogenic than a PCV-13 booster in PCV-13 primed children. Post booster antibody responses that were similar or superior in the PCV-10 group were seen for serotypes 4, $18 \mathrm{C}$ and 19F, which are contained in PCV-10 with higher antigen content and/or conjugation to a diphtheria or tetanus toxoid carrier protein. The clinical significance of the observed differences in immunogenicity remains unknown although our findings suggest that it may be more appropriate to give a PCV-13 booster to PCV-13 primed children. Further studies in children primed with PCV-10 and then boosted with PCV-13 are needed to expand our understanding and the clinical implications of interchangeable PCV schedules.

\section{ACKNOWLEDGMENTS}

The authors are grateful to all the study participants, without whom this study would have not been possible. A.J.P. and M.D.S. are Jenner Institute Investigators. We thank Dominic F. Kelly for his critical review of the manuscript.

\section{REFERENCES}

1. Miller E, Andrews NJ, Waight PA, et al. Herd immunity and serotype replacement 4 years after seven-valent pneumococcal conjugate vaccination in England and Wales: an observational cohort study. Lancet Infect Dis. 2011;11:760-768.

2. Pilishvili T, Lexau C, Farley MM, et al.; Active Bacterial Core Surveillance/ Emerging Infections Program Network. Sustained reductions in invasive pneumococcal disease in the era of conjugate vaccine. $J$ Infect Dis. 2010;201:32-41.

3. Moore CE, Paul J, Foster D, et al.; Oxford Invasive Pneumococcal Surveillance Group. Reduction of invasive pneumococcal disease 3 years after the introduction of the 13-valent conjugate vaccine in the Oxfordshire region of England. J Infect Dis. 2014;210:1001-1011.

4. Deceuninck G, De Serres G, Boulianne N, et al. Effectiveness of three pneumococcal conjugate vaccines to prevent invasive pneumococcal disease in Quebec, Canada. Vaccine. 2015;33:2684-2689.

5. Palmu AA, Jokinen J, Borys D, et al. Effectiveness of the ten-valent pneumococcal Haemophilus influenzae protein D conjugate vaccine (PHiD-CV10) against invasive pneumococcal disease: a cluster randomised trial. Lancet. 2013;381:214-222.
6. Waight PA, Andrews NJ, Ladhani SN, et al. Effect of the 13-valent pneumococcal conjugate vaccine on invasive pneumococcal disease in England and Wales 4 years after its introduction: an observational cohort study. Lancet Infect Dis. 2015;15:535-543

7. Poolman JT, Peeters CC, van den Dobbelsteen GP. The history of pneumococcal conjugate vaccine development: dose selection. Expert Rev Vaccines. 2013;12:1379-1394.

8. Dagan R, Poolman J, Siegrist CA. Glycoconjugate vaccines and immune interference: a review. Vaccine. 2010;28:5513-5523.

9. Prymula R, Peeters P, Chrobok V, et al. Pneumococcal capsular polysaccharides conjugated to protein $\mathrm{D}$ for prevention of acute otitis media caused by both Streptococcus pneumoniae and non-typable Haemophilus influenzae: a randomised double-blind efficacy study. Lancet. 2006;367:740-748.

10. Tregnaghi MW, Sáez-Llorens X, López P, et al.; COMPAS Group. Efficacy of pneumococcal nontypable Haemophilus influenzae protein D conjugate vaccine (PHiD-CV) in young Latin American children: a double-blind randomized controlled trial. PLoS Med. 2014;11:e1001657.

11. Rappuoli R, De Gregorio E. A sweet $\mathrm{T}$ cell response. Nat Med. 2011;17:1551-1552.

12. Rose CE, Romero-Steiner S, Burton RL, et al. Multilaboratory comparison of Streptococcus pneumoniae opsonophagocytic killing assays and their level of agreement for the determination of functional antibody activity in human reference sera. Clin Vaccine Immunol. 2011;18:135-142.

13. R Foundation. The R project for statistical computing. 2016;3.

14. Siber GR, Chang I, Baker S, et al. Estimating the protective concentration of anti-pneumococcal capsular polysaccharide antibodies. Vaccine. 2007;25:3816-3826

15. Snape MD, Klinger CL, Daniels ED, et al. Immunogenicity and reactogenicity of a 13-valent-pneumococcal conjugate vaccine administered at 2,4 , and 12 months of age: a double-blind randomized active-controlled trial. Pediatr Infect Dis J. 2010;29:e80-e90.

16. Dagan R, Patterson S, Juergens C, et al. Comparative immunogenicity and efficacy of 13 -valent and 7-valent pneumococcal conjugate vaccines in reducing nasopharyngeal colonization: a randomized double-blind trial. Clin Infect Dis. 2013;57:952-962.

17. Andrews NJ, Waight PA, Burbidge P, et al. Serotype-specific effectiveness and correlates of protection for the 13-valent pneumococcal conjugate vaccine: postlicensure indirect cohort study. Lancet Infect Dis. 2014;14:839-846.

18. Trück J, Pollard AJ. Challenges in immunisation against bacterial infection in children. Early Hum Dev. 2010;86:695-701

19. van Westen E, Wijmenga-Monsuur AJ, van Dijken HH, et al. Differential B-cell memory around the 11-month booster in children vaccinated with a 10- or 13-valent pneumococcal conjugate vaccine. Clin Infect Dis. 2015;61:342-349

20. Wijmenga-Monsuur AJ, van Westen E, Knol MJ, et al. Direct comparison of immunogenicity induced by 10 - or 13-valent pneumococcal conjugate vaccine around the 11-month booster in Dutch infants. PLoS One 2015;10:e0144739.

21. Malito E, Bursulaya B, Chen C, et al. Structural basis for lack of toxicity of the diphtheria toxin mutant CRM197. Proc Natl Acad Sci USA. 2012;109:5229-5234.

22. Vesikari T, Wysocki J, Chevallier B, et al. Immunogenicity of the 10-valent pneumococcal non-typeable Haemophilus influenzae protein D conjugate vaccine (PHiD-CV) compared to the licensed 7vCRM vaccine. Pediatr Infect Dis J. 2009;28(4 suppl):S66-S76.

23. Russell FM, Carapetis JR, Balloch A, et al. Hyporesponsiveness to re-challenge dose following pneumococcal polysaccharide vaccine at 12 months of age, a randomized controlled trial. Vaccine. 2010;28:3341-3349.

24. Trück J, Snape MD, Tatangeli F, et al. Pneumococcal serotype-specific antibodies persist through early childhood after infant immunization: follow-up from a randomized controlled trial. PLoS One. 2014;9:e91413. 